\title{
Lie Group Solutions of Magnetohydrodynamics Equations and Their Well-Posedness
}

\author{
Fu-zhi Li, ${ }^{1}$ Jia-li Yu, ${ }^{2}$ Yang-rong Li, ${ }^{1}$ and Gan-shan Yang ${ }^{3}$ \\ ${ }^{1}$ School of Mathematics and Statistics, Southwest University, Chongqing 400715, China \\ ${ }^{2}$ School of Mathematics and Information Science, Guangzhou University, Guangzhou 510006, China \\ ${ }^{3}$ School of Mathematics and Computer Science, Yunnan Minzu University, Kunming, Yunnan 650500, China
}

Correspondence should be addressed to Gan-shan Yang; yganshan@aliyun.com

Received 27 June 2016; Accepted 20 September 2016

Academic Editor: Bingwen Liu

Copyright (C) $2016 \mathrm{Fu}$-zhi Li et al. This is an open access article distributed under the Creative Commons Attribution License, which permits unrestricted use, distribution, and reproduction in any medium, provided the original work is properly cited.

Based on classical Lie Group method, we construct a class of explicit solutions of two-dimensional ideal incompressible magnetohydrodynamics (MHD) equation by its infinitesimal generator. Via these explicit solutions we study the uniqueness and stability of initial-boundary problem on MHD.

\section{Introduction}

Two-dimensional ideal incompressible magnetohydrodynamics (MHD) equation can be described by a set of two scalar equations for the vorticity $w$ and the magnetic stream function $\psi$; namely [1],

$$
\begin{aligned}
& \left(\partial_{t}+u \cdot \nabla\right) w=B \cdot \nabla j, \\
& \left(\partial_{t}+u \cdot \nabla\right) \psi=0 .
\end{aligned}
$$

Due to the divergence freedom of the magnetic field $B$, it is possible to define a magnetic stream function $\psi$ via $B=\nabla^{\perp} \psi$. In the incompressible case, $\nabla \cdot u=0$, the velocity stream function $\varphi$ and velocity $u$ are connected in the same way, and $u=\nabla^{\perp} \varphi$. Vorticity and current density are defined as the Laplacian of the stream functions, $w=\Delta \varphi$ and $j=\Delta \varphi$. The magnetic stream function is convected with the flow field [2-4]; that means the ideal MHD equations do not allow for magnetic reconnection in contrast to the dissipative version of the above equations [5].

Note that in contrast to the two-dimensional incompressible Euler equations case, there is not a production term on the right-hand side of (1). The equations show a tendency to develop fine structures, namely, current sheets. Analytically the problem about the regularity of solutions is still an open problem $[6,7]$.
It is interesting to seek the solutions of MHD in mathematics and physics for a long time [8]. In order to construct solutions of MHD, many effective methods have been put forward, such as the inverse scattering method, Backlund transformation, Hirota method, and homogeneous balance method [3]. In the branches of mathematics and physics, Lie Group theory [9-11] was often used extensively. Ever since the 1970s Bluman and Col proposed similarity theory for differential equations, the Lie Group theory has been developed indifferential equations. The main idea of Lie Group method is to use the prolongation formulae, providing an effective computational procedure for finding the most general symmetry group of almost any system of partial differential equations of interest. To the best of our knowledge, related classical Lie Group method has not been preformed to the MHD equation.

Many mathematicians are devoted to studying the MHD equations. For example, Duvant and Lions proved the existence and uniqueness of the global strong solutions of twodimensional MHD equations with initial-boundary value problem. They also proved existence and uniqueness of locally strong solutions and the existence of the global weak solutions of three-dimensional MHD equations $[6,12]$. As we all know, the studying of uniqueness and stability of MHD equations is based on some certain conditions or some assumptions. But, in this paper, we study a special class of 
solutions-wave solutions. It does not need external hypotheses when proving the uniqueness, which is a novelty of this paper.

\section{Calculation of Symmetry Groups}

In this section, we perform Lie symmetry analysis for (1) and

(2) and obtain its infinitesimal generator. Now we transform

(1) and (2) as follows:

$$
\begin{gathered}
\frac{\partial^{3} \varphi}{\partial x_{1}^{2} \partial t}+\frac{\partial^{3} \varphi}{\partial x_{2}^{2} \partial t}+\frac{\partial \varphi}{\partial x_{1}} \frac{\partial^{3} \varphi}{\partial x_{2}^{3}}+\frac{\partial \varphi}{\partial x_{1}} \frac{\partial^{3} \varphi}{\partial x_{1}^{2} \partial x_{2}}-\frac{\partial \varphi}{\partial x_{2}} \frac{\partial^{3} \varphi}{\partial x_{1}^{3}} \\
-\frac{\partial \varphi}{\partial x_{2}} \frac{\partial^{3} \varphi}{\partial x_{2}^{2} \partial x_{1}}=\frac{\partial \psi}{\partial x_{1}} \frac{\partial^{3} \psi}{\partial x_{2}^{3}}+\frac{\partial \psi}{\partial x_{1}} \frac{\partial^{3} \psi}{\partial x_{1}^{2} \partial x_{2}} \\
-\frac{\partial \psi}{\partial x_{2}} \frac{\partial^{3} \psi}{\partial x_{1}^{3}}-\frac{\partial \psi}{\partial x_{2}} \frac{\partial^{3} \psi}{\partial x_{2}^{2} \partial x_{1}} \\
\frac{\partial \psi}{\partial t}+\frac{\partial \varphi}{\partial x_{1}} \frac{\partial \psi}{\partial x_{2}}-\frac{\partial \psi}{\partial x_{1}} \frac{\partial \varphi}{\partial x_{2}}=0 .
\end{gathered}
$$

According to the method of determining the infinitesimal generator of nonlinear partial differential equation, we take the infinitesimal generator of equation as follows:

$$
v=\sum_{i=1}^{p} \xi^{i}(x, u) \frac{\partial}{\partial x^{i}}+\sum_{\alpha=1}^{q} \phi_{\alpha}(x, u) \frac{\partial}{\partial u^{\alpha}} .
$$

It is a vector field defined on an open subset $M \subset X \times U$; the $n$th prolongation of $v$ is the vector field

$$
\operatorname{pr}^{(n)} v=v+\sum_{\alpha=1}^{q} \sum_{J} \phi_{\alpha}^{J}\left(x, u^{(n)}\right) \frac{\partial}{\partial u_{J}^{\alpha}}
$$

defined on the corresponding jet space $M^{(n)} \subset X \times U^{(n)}$, the second summation being overall multi-indices $J=$ $\left(j_{1}, j_{2}, j_{3}, \ldots, j_{k}\right)$, with $1 \leq j_{k} \leq p, 1 \leq k \leq n$. The coefficient functions $\phi_{\alpha}^{J}$ of $\operatorname{pr}^{(n)} v$ are given by the following formula:

$$
\phi_{\alpha}^{J}\left(x, u^{(n)}\right)=D_{J}\left(\phi_{\alpha}-\sum_{i=1}^{p} \xi^{i} u_{i}^{\alpha}\right)+\sum_{i=1}^{p} \xi^{i} u_{J, i}^{\alpha}
$$

where $u_{i}^{\alpha}=\partial u^{\alpha} / \partial x^{i}$ and $u_{J, i}^{\alpha}=\partial u_{J}^{\alpha} / \partial x^{i}$ (in this paper $x^{1}=$ $x_{1}, x^{2}=x_{2}$, and $x^{3}=t$ ).

Firstly, we consider the circumstance of $n=1$. Let

$$
v=\xi_{1} \frac{\partial}{\partial x_{1}}+\xi_{2} \frac{\partial}{\partial x_{2}}+\xi_{3} \frac{\partial}{\partial t}+\xi_{4} \frac{\partial}{\partial \varphi}+\xi_{5} \frac{\partial}{\partial \psi},
$$

where $\xi_{i}=\xi_{i}\left(x_{1}, x_{2}, t, \varphi, \psi\right)(i=1,2,3,4)$ are coefficient functions of the infinitesimal generator to be determined. And the first-order prolongation of $v$ is as follows:

$$
\begin{aligned}
\operatorname{pr}^{(1)} v= & v+\eta_{1}^{x_{1}} \frac{\partial}{\partial \varphi_{x_{1}}}+\eta_{2}^{x_{2}} \frac{\partial}{\partial \varphi_{x_{2}}}+\eta_{3}^{x_{1}} \frac{\partial}{\partial \psi_{x_{1}}}+\eta_{4}^{x_{2}} \frac{\partial}{\partial \psi_{x_{2}}} \\
& +\eta_{5}^{t} \frac{\partial}{\partial \varphi_{t}}+\eta_{6}^{t} \frac{\partial}{\partial \psi_{t}} .
\end{aligned}
$$

Applying $\operatorname{pr}^{(1)} v$ to (3), we find

$$
\begin{aligned}
& \left(\frac{\partial^{3} \varphi}{\partial x_{2}^{3}}+\frac{\partial^{3} \varphi}{\partial x_{1}^{2} \partial x_{2}}\right) \eta_{1}^{x_{1}}-\left(\frac{\partial^{3} \varphi}{\partial x_{1}^{3}}+\frac{\partial^{3} \varphi}{\partial x_{2}^{2} \partial x_{1}}\right) \eta_{2}^{x_{2}} \\
& -\left(\frac{\partial^{3} \psi}{\partial x_{2}^{3}}+\frac{\partial^{3} \psi}{\partial x_{1}^{2} \partial x_{2}}\right) \eta_{3}^{x_{1}} \\
& -\left(\frac{\partial^{3} \psi}{\partial x_{1}^{3}}+\frac{\partial^{3} \psi}{\partial x_{2}^{2} \partial x_{1}}\right) \eta_{4}^{x_{2}}=0, \\
& \frac{\partial \psi}{\partial x_{2}} \eta_{1}^{x_{1}}-\frac{\partial \psi}{\partial x_{1}} \eta_{2}^{x_{2}}-\frac{\partial \varphi}{\partial x_{2}} \eta_{3}^{x_{1}}+\frac{\partial \varphi}{\partial x_{1}} \eta_{2}^{x_{4}}+\eta_{6}^{t}=0 .
\end{aligned}
$$

Now we apply the third-order prolongation of $v$ to (1) and (2). Let

$$
\begin{aligned}
v & =\sum_{i=1}^{3} \xi^{i}(x, u) \frac{\partial}{\partial x^{i}}+\sum_{\alpha=1}^{2} \phi_{\alpha}(x, u) \frac{\partial}{\partial u^{\alpha}} \\
\operatorname{pr}^{(3)} v & =v+\sum_{\alpha=1}^{q} \sum_{J} \phi_{\alpha}^{J}\left(x, u^{(3)}\right) \frac{\partial}{\partial u_{J}^{\alpha}},
\end{aligned}
$$

where $q=2, u^{1}=\varphi$, and $u^{2}=\psi$, the second summation being overall multi-indices $J=\left(j_{1}, j_{2}, j_{3}, \ldots, j_{k}\right)$, with $1 \leq$ $j_{k} \leq 3,1 \leq k \leq 3$.

The coefficient functions $\phi_{\alpha}^{J}$ of $\mathrm{pr}^{(3)} v$ are given by the following formula:

$$
\phi_{\alpha}^{J}\left(x, u^{(3)}\right)=D_{J}\left(\phi_{\alpha}-\sum_{i=1}^{3} \xi^{i} u_{i}^{\alpha}\right)+\sum_{i=1}^{3} \xi^{i} u_{J, i}^{\alpha}
$$

where $u_{i}^{\alpha}=\partial u^{\alpha} / \partial x^{i}$ and $u_{J, i}^{\alpha}=\partial u_{J}^{\alpha} / \partial x^{i}\left(x^{1}=x_{1}, x^{2}=x_{2}\right.$, $\left.x^{3}=t\right)$ :

$$
\begin{aligned}
\operatorname{pr}^{(3)} v= & v+\eta_{1}^{x_{1}} \frac{\partial}{\partial \varphi_{x_{1}}}+\eta_{2}^{x_{2}} \frac{\partial}{\partial \varphi_{x_{2}}}+\eta_{3}^{x_{1}} \frac{\partial}{\partial \psi_{x_{1}}} \\
& +\eta_{4}^{x_{2}} \frac{\partial}{\partial \psi_{x_{2}}}+\eta_{5}^{t} \frac{\partial}{\partial \varphi_{t}}+\eta_{6}^{t} \frac{\partial}{\partial \psi_{t}}+\phi_{1}^{x_{1} x_{1} t} \frac{\partial}{\partial \varphi_{x_{1} x_{1} t}} \\
& +\phi_{1}^{x_{2} x_{2} t} \frac{\partial}{\partial \varphi_{x_{2} x_{2} t}}+\phi_{1}^{x_{2} x_{2} x_{2}} \frac{\partial}{\partial \varphi_{x_{2} x_{2} x_{2}}} \\
& +\phi_{1}^{x_{1} x_{2} x_{2}} \frac{\partial}{\partial \varphi_{x_{1} x_{2} x_{2}}} \\
& +\phi_{1}^{x_{1} x_{1} x_{2}} \frac{\partial}{\partial \varphi_{x_{1} x_{1} x_{2}}} \phi_{1}^{x_{1} x_{1} x_{1}} \frac{\partial}{\partial \varphi_{x_{1} x_{1} x_{1}}} \\
& +\phi_{2}^{x_{1} x_{1} x_{1}} \frac{\partial}{\partial \psi_{x_{1} x_{1} x_{2}}}+\phi_{2}^{x_{1} x_{1} x_{1}} \frac{\partial}{\partial \psi_{x_{1} x_{1} x_{2}}} \\
& +\phi_{2}^{x_{1} x_{2} x_{2}} \frac{\partial}{\partial \psi_{x_{1} x_{2} x_{2}}}+\phi_{2}^{x_{2} x_{2} x_{2}} \frac{\partial}{\partial \psi_{x_{2} x_{2} x_{2}}} .
\end{aligned}
$$


Applying $\operatorname{pr}^{(3)} v$ to (3), we find

$$
\begin{gathered}
\phi_{1}^{x_{1} x_{1} t}+\phi_{1}^{x_{2} x_{2} t}+\phi_{1}^{x_{2} x_{2} x_{2}} \frac{\partial \varphi}{\partial x_{1}}+\phi_{1}^{x_{1} x_{1} x_{2}} \frac{\partial \varphi}{\partial x_{1}} \\
-\phi_{1}^{x_{1} x_{1} x_{1}} \frac{\partial \varphi}{\partial x_{2}}-\phi_{1}^{x_{1} x_{2} x_{2}} \frac{\partial \varphi}{\partial x_{2}}+\eta_{1}^{x_{1}} \frac{\partial^{3} \varphi}{\partial x_{2}^{3}}-\eta_{1}^{x_{2}} \frac{\partial^{3} \varphi}{\partial x_{1}^{3}} \\
+\eta_{1}^{x_{1}} \frac{\partial^{3} \varphi}{\partial x_{1}^{2} \partial x_{2}}-\eta_{2}^{x_{2}} \frac{\partial^{3} \varphi}{\partial x_{2}^{2} \partial x_{1}}=\phi_{2}^{x_{2} x_{2} x_{2}} \frac{\partial \psi}{\partial x_{1}} \\
+\phi_{2}^{x_{1} x_{1} x_{2}} \frac{\partial \psi}{\partial x_{1}}-\phi_{2}^{x_{1} x_{1} x_{1}} \frac{\partial \psi}{\partial x_{2}}-\phi_{2}^{x_{1} x_{2} x_{2}} \frac{\partial \psi}{\partial x_{2}} \\
+\eta_{3}^{x_{1}} \frac{\partial^{3} \psi}{\partial x_{2}^{3}}-\eta_{4}^{x_{2}} \frac{\partial^{3} \psi}{\partial x_{1}^{3}}+\eta_{3}^{x_{1}} \frac{\partial^{3} \psi}{\partial x_{1}^{2} \partial x_{2}}-\eta_{4}^{x_{2}} \frac{\partial^{3} \psi}{\partial x_{2}^{2} \partial x_{1}} \\
\frac{\partial \psi}{\partial x_{2}} \eta_{1}^{x_{1}}-\frac{\partial \psi}{\partial x_{1}} \eta_{2}^{x_{2}}-\frac{\partial \varphi}{\partial x_{2}} \eta_{3}^{x_{1}}+\frac{\partial \varphi}{\partial x_{1}} \eta_{2}^{x_{4}}+\eta_{6}^{t}=0 .
\end{gathered}
$$

Then merging similar terms coefficients, we have

$$
\eta_{1}^{x_{1}}=\eta_{2}^{x_{2}}=\eta_{3}^{x_{1}}=\eta_{4}^{x_{2}}=\eta_{6}^{t}=0 .
$$

According to the formula $\phi_{\alpha}^{J}\left(x, u^{(n)}\right)=D_{J}\left(\phi_{\alpha}-\sum_{i=1}^{p} \xi^{i} u_{i}^{\alpha}\right)+$ $\sum_{i=1}^{p} \xi^{i} u_{J, i}^{\alpha}$, for example, we have

$$
\begin{aligned}
\eta_{6}^{t}= & D_{t}\left(\xi_{5}-\xi_{1} \psi_{x_{1}}-\xi_{2} \psi_{x_{2}}-\xi_{3} \psi_{t}\right)+\xi_{1} \psi_{x_{1} t} \\
& +\xi_{2} \psi_{x_{2} t}+\xi_{3} \psi_{t t} \\
= & \xi_{5 t}-\xi_{1 t} \psi_{x_{1}}-\xi_{1 \psi} \psi_{x_{1}} \psi_{t}-\xi_{1 \varphi} \psi_{x_{1}} \psi_{t}-\xi_{2 t} \psi_{x_{2}} \\
& -\xi_{2 \psi} \psi_{x_{2}} \psi_{t}-\xi_{2 \varphi} \psi_{x_{2}} \varphi_{t}-\xi_{3} t \psi_{t}-\xi_{3 \varphi} \varphi_{t} \psi_{t} \\
& -\xi_{3 \psi} \psi_{t} \psi_{t}+\xi_{5 \varphi} \varphi_{t}+\xi_{5 \psi} \psi_{t} \\
\phi_{1}^{x_{1} x_{1} x_{1}}= & D_{x_{1}}^{3}\left(\xi_{4}-\xi_{1} \varphi_{x_{1}}-\xi_{2} \varphi_{x_{2}}-\xi_{3} \varphi_{t}\right) \\
& +\xi_{1} \varphi_{x_{1} x_{1} x_{1} x_{1}}+\xi_{2} \varphi_{x_{1} x_{1} x_{1} x_{2}}+\xi_{3} \varphi_{x_{1} x_{1} x_{1} t} \\
= & D_{x_{1}}^{3} \xi_{4}-D_{x_{1}}^{3} \xi_{1} \varphi_{x_{1}} D_{x_{1}}^{3} \xi_{2} \varphi_{x_{2}}-D_{x_{1}}^{3} \xi_{3} \varphi_{t} \\
& -3 D_{x_{1}}^{2} \xi_{1} \varphi_{x_{1} x_{1}}-3 D_{x_{1}}^{2} \xi_{2} \varphi_{x_{1} x_{2}}-3 D_{x_{1}}^{2} \xi_{3} \varphi_{x_{1} t} \\
& -3 D_{x_{1}} \xi_{1} \varphi_{x_{1} x_{1} x_{1}}-3 D_{x_{1}} \xi_{2} \varphi_{x_{1} x_{1} x_{2}} \\
& -3 D_{x_{1}} \xi_{3} \varphi_{x_{1} x_{1} t} .
\end{aligned}
$$

So we have

$$
\begin{aligned}
\xi_{5 t} & =0, \\
\xi_{5 \psi}-\xi_{3 t} & =0, \\
\xi_{1 t} & =\xi_{1 \varphi}=\xi_{1 \psi}=\xi_{2 t}=\xi_{2 \varphi}=\xi_{2 \psi}=\xi_{3 \varphi}=\xi_{3 \psi} \\
& =\xi_{5 \varphi}=0 .
\end{aligned}
$$

Similarly we can find the determining equations for the symmetry group of the equations to be the following:

$$
\begin{aligned}
\xi_{4 x_{1}} & =0, \\
\xi_{4 \varphi}-\xi_{1 x_{1}} & =0, \\
\xi_{1 \varphi} & =\xi_{1 \psi}=\xi_{2 \varphi}=\xi_{2 \psi}=\xi_{2 x_{1}}=\xi_{3 x_{1}}=\xi_{3 \varphi} \\
& =\xi_{3 \psi}=\xi_{5 \psi}=0 . \\
\xi_{4 x_{2}} & =0, \\
\xi_{4 \varphi}-\xi_{2 x_{2}} & =0, \\
\xi_{1 \varphi} & =\xi_{1 \psi}=\xi_{1 x_{2}}=\xi_{2 \varphi}=\xi_{2 \psi}=\xi_{3 x_{2}}=\xi_{3 \varphi} \\
& =\xi_{3 \psi}=\xi_{4 \psi}=0 . \\
\xi_{5 x_{1}} & =0, \\
\xi_{5 \psi}-\xi_{1 x_{1}} & =0, \\
\xi_{1 \varphi} & =\xi_{1 \psi}=\xi_{2 \varphi}=\xi_{2 \psi}=\xi_{2 x_{1}}=\xi_{3 x_{1}}=\xi_{3 \varphi} \\
& =\xi_{3 \psi}=\xi_{5 \varphi}=0 . \\
\xi_{5 x_{2}} & =0, \\
\xi_{5 \psi}-\xi_{2 x_{2}} & =0, \\
\xi_{1 \varphi} & =\xi_{1 \psi}=\xi_{1 x_{2}}=\xi_{2 \varphi}=\xi_{2 \psi}=\xi_{3 x_{2}}=\xi_{3 \varphi} \\
& =\xi_{3 \psi}=\xi_{5 \varphi}=0 .
\end{aligned}
$$

As usual, subscripts indicate derivatives. The solution of the determining equations is elementary. According to (16) and (17) we have

$$
\begin{aligned}
& \xi_{4}=\xi_{4}(t, \varphi), \\
& \xi_{5}=\xi_{5}(\psi), \\
& \xi_{1}=\xi_{1}\left(x_{1}\right), \\
& \xi_{2}=\xi_{2}\left(x_{2}\right), \\
& \xi_{3}=\xi_{3}(t), \\
& \xi_{3 t}=\xi_{5 \psi}, \\
& \xi_{4 \varphi}=\xi_{1 x_{1}}, \\
& \xi_{4 \varphi}=\xi_{2 x_{2}}, \\
& \xi_{5 \psi}=\xi_{1 x_{1}}, \\
& \xi_{5 \psi}=\xi_{2 x_{2}} .
\end{aligned}
$$

Finally, solving the above differential equations, we conclude that the most general infinitesimal symmetry of (3) has coefficient functions of the form

$$
\begin{aligned}
& \xi_{1}=k x_{1}+a \\
& \xi_{2}=k x_{2}+b
\end{aligned}
$$




$$
\begin{aligned}
& \xi_{3}=k t+c \\
& \xi_{4}=k \varphi+\sigma(t)+d \\
& \xi_{5}=k \psi+e,
\end{aligned}
$$

where $a, b, c, d, e$, and $k$ are arbitrary constants and $\sigma(t)$ is an arbitrary function of $t$ only. Thus the Lie algebra of infinitesimal symmetries of the MHD equations is spanned by the six vector fields:

$$
\begin{aligned}
& v_{1}=x_{1} \partial_{x_{1}}+x_{2} \partial_{x_{2}}+t \partial_{t}+\varphi \partial_{\varphi}+\psi \partial_{\psi} \\
& v_{2}=\partial_{x_{1}} \\
& v_{3}=\partial_{x_{2}} \\
& v_{4}=\partial_{t} \\
& v_{5}=\partial_{\varphi} \\
& v_{6}=\partial_{\psi} .
\end{aligned}
$$

And the infinite-dimensional subalgebra

$$
v_{\alpha}=\sigma(t) \partial_{\varphi}
$$

where $\sigma(t)$ is an arbitrary function of $t$ only. So we have

$$
v=c_{1} v_{1}+c_{2} v_{2}+c_{3} v_{3}+c_{4} v_{4}+c_{5} v_{5}+c_{6} v_{6}+v_{\alpha} .
$$

The entries give the transformed point $\exp \left(\epsilon_{i}\right)\left(x_{1}, x_{2}, t\right.$, $\varphi, \psi)=\left(\widetilde{x_{1}}, \widetilde{x_{2}}, \widetilde{t}, \widetilde{\varphi}, \widetilde{\psi}\right)$ :

$$
\begin{gathered}
G_{1}:\left(e^{\epsilon} x_{1}, e^{\epsilon} x_{2}, e^{\epsilon} t, e^{\epsilon} \varphi, e^{\epsilon} \psi\right) \\
G_{2}:\left(x_{1}+\epsilon, x_{2}, t, \varphi, \psi\right) \\
G_{3}:\left(x_{1}, x_{2}+\epsilon, t, \varphi, \psi\right) \\
G_{4}:\left(x_{1}, x_{2}, t+\epsilon, \varphi, \psi\right) \\
G_{5}:\left(x_{1}, x_{2}, t, \varphi+\epsilon, \psi\right) \\
G_{6}:\left(x_{1}, x_{2}, t, \varphi, \psi+\epsilon\right) \\
G_{\alpha}:\left(x_{1}, x_{2}, t, \varphi+\epsilon \sigma(t), \psi\right) .
\end{gathered}
$$

If $\varphi=f\left(x_{1}, x_{2}, t\right)$ and $\psi=g\left(x_{1}, x_{2}, t\right)$ are known solutions of (3), then using the above groups $G_{i}, i=(1,2, \ldots, 6)$, the corresponding new solutions $\varphi_{i}$ and $\psi_{i}$ can be obtained, respectively, as follows:

$$
\begin{aligned}
& \varphi_{1}=e^{\epsilon} f\left(e^{-\epsilon} x_{1}, e^{-\epsilon} x_{2}, e^{-\epsilon} t\right), \\
& \psi_{1}=e^{\epsilon} g\left(e^{-\epsilon} x_{1}, e^{-\epsilon} x_{2}, e^{-\epsilon} t\right), \\
& \varphi_{2}=f\left(x_{1}-\epsilon, x_{2}, t\right), \\
& \psi_{2}=g\left(x_{1}-\epsilon, x_{2}, t\right), \\
& \varphi_{3}=f\left(x_{1}, x_{2}-\epsilon, t\right), \\
& \psi_{3}=g\left(x_{1}, x_{2}-\epsilon, t\right),
\end{aligned}
$$

$$
\begin{aligned}
& \varphi_{4}=f\left(x_{1}, x_{2}, t-\epsilon\right), \\
& \psi_{4}=g\left(x_{1}, x_{2}, t-\epsilon\right), \\
& \varphi_{5}=f\left(x_{1}, x_{2}, t\right)+\epsilon, \\
& \psi_{5}=g\left(x_{1}, x_{2}, t\right), \\
& \varphi_{6}=f\left(x_{1}, x_{2}, t\right), \\
& \psi_{6}=g\left(x_{1}, x_{2}, t\right)+\epsilon, \\
& \varphi_{\alpha}=f\left(x_{1}, x_{2}, t\right)+\epsilon \sigma(t), \\
& \psi_{\alpha}=g\left(x_{1}, x_{2}, t\right),
\end{aligned}
$$

where $\epsilon$ is a real number and $\sigma(t)$ is an arbitrary function of $t$ only.

Theorem 1. For the known solutions $\varphi=f\left(x_{1}, x_{2}, t\right)$ and $\psi=$ $g\left(x_{1}, x_{2}, t\right)$, by using one-parameter groups $G_{i}, i=(1,2, \ldots, 6)$ continuously, one can obtain a new solution which can be expressed in the following form:

$$
\begin{aligned}
\varphi= & e^{\epsilon_{1}} f\left(e^{-\epsilon} x_{1}-\epsilon_{2}, e^{-\epsilon} x_{2}-\epsilon_{3},-e^{-\epsilon} t-\epsilon_{4}\right)+\epsilon_{5} \\
& +\epsilon_{6} \sigma(t) \\
\psi= & e^{\epsilon_{1}} f\left(e^{-\epsilon} x_{1}-\epsilon_{2}, e^{-\epsilon} x_{2}-\epsilon_{3},-e^{-\epsilon} t-\epsilon_{4}\right)+\epsilon_{5},
\end{aligned}
$$

where $\epsilon_{i}, i=(1,2, \ldots, 6)$ are arbitrary constants.

Theorem 2. One assumes that the solutions have forms of $\varphi=$ $\varphi\left(a_{1} x_{1}+b_{1} x_{2}+c_{1} t+d_{1}\right), \psi=\psi\left(a_{2} x_{1}+b_{2} x_{2}+c_{2} t+d_{2}\right)$.

One has the following:

(1) If $c_{1}=c_{2}=0, a_{1} b_{2}-a_{2} b_{1}=0$, then $\varphi, \psi$ are arbitrary functions.

(2) If $a_{1}=b_{1}=c_{2}=0$ and $c_{1}, a_{2}=b_{2}$ are arbitrary constants, then $\varphi, \psi$ are arbitrary functions.

(3) If $\varphi=-\left(c_{2} /\left(a_{1} b_{2}-a_{2} b_{1}\right)\right)\left(a_{1} x_{1}+b_{1} x_{2}+c_{1} t+d_{1}\right)$, then $\psi$ is arbitrary function.

\section{The Uniqueness of Wave Solutions}

In this section we give some nonzero solutions by considering the wave solutions to the two-dimensional MHD equations [13-15]. Firstly we could give the initial-boundary value of three conditions in Theorem 2.

(1) $\left.\varphi\left(x_{1}, x_{2}, t\right)\right|_{t=0}=\varphi\left(a_{1} x_{1}+b_{1} x_{2}+d_{1}\right) \in H_{0}^{1}(\Omega)$, $\left.\varphi\left(x_{1}, x_{2}, t\right)\right|_{x \in \partial \Omega}=0$ $\left.\psi\left(x_{1}, x_{2}, t\right)\right|_{t=0}=\psi\left(k a_{1} x_{1}+k b_{1} x_{2}+d_{1}\right) \in H_{0}^{1}(\Omega)$, $\left.\psi\left(x_{1}, x_{2}, t\right)\right|_{x \in \partial \Omega}=0$.

(2) $\left.\varphi\left(x_{1}, x_{2}, t\right)\right|_{t=0}=\varphi\left(d_{1}\right) \in H_{0}^{1}(\Omega),\left.\varphi\left(x_{1}, x_{2}, t\right)\right|_{x \in \partial \Omega}=$ 0 $\left.\psi\left(x_{1}, x_{2}, t\right)\right|_{t=0}=\psi\left(a_{2} x_{1}+b_{2} x_{2}+d_{1}\right) \in H_{0}^{1}(\Omega)$, $\left.\psi\left(x_{1}, x_{2}, t\right)\right|_{x \in \partial \Omega}=0$.

(3) $\left.\varphi\left(x_{1}, x_{2}, t\right)\right|_{t=0}=-\left(c_{2} /\left(a_{1} b_{2}-a_{2} b_{1}\right)\right)\left(a_{1} x_{1}+b_{1} x_{2}+d_{1}\right) \epsilon$ $H_{0}^{1}(\Omega),\left.\varphi\left(x_{1}, x_{2}, t\right)\right|_{x \in \partial \Omega}=0$.

$\left.\psi\left(x_{1}, x_{2}, t\right)\right|_{t=0} \in H_{0}^{1}(\Omega),\left.\psi\left(x_{1}, x_{2}, t\right)\right|_{x \in \partial \Omega}=0$. 
Theorem 3. Assume that $\Omega \subset R^{2}$ is a bounded domain. One can make the solutions $\varphi_{1}, \psi_{1}$ like the condition in Theorem 2 and satisfying $\left\|\Delta \psi_{1}\right\|_{L^{\infty}},\left\|\nabla \psi_{1}\right\|_{L^{\infty}},\left\|D^{2} \varphi_{1}\right\|_{L^{\infty}} \leq M$, respectively. Then the following initial-boundary value problem:

$$
\begin{array}{rlrl}
\left(\partial_{t}+u \cdot \nabla\right) w & =B \cdot \nabla j, \quad \text { in } \Omega \times(0, \infty), \\
\left(\partial_{t}+u \cdot \nabla\right) \psi & =0, \quad \text { in } \Omega \times(0, \infty), \\
\varphi & =\varphi_{0}\left(a_{1} x_{1}+b_{1} x_{2}+d_{1}\right), & \text { in } \Omega \times 0, \\
\psi & =\psi_{0}\left(a_{2} x_{1}+b_{2} x_{2}+d_{2}\right), & \text { in } \Omega \times 0
\end{array}
$$

has a unique smooth solution $\varphi$, $\psi$.

Proof. To prove the uniqueness we consider two smooth solution pairs, say $\varphi_{1}, \psi_{1}$ and $\varphi, \psi$. Let their difference be $\bar{\varphi}=\varphi-\varphi_{1}, \bar{\psi}=\psi-\psi_{1}$. Then subtracting the equations from each other in (26), we have

$$
\begin{aligned}
& \Delta \overline{\varphi_{t}}+\nabla^{\perp} \varphi \cdot \nabla \Delta \varphi-\nabla^{\perp} \varphi_{1} \cdot \nabla \Delta \varphi_{1} \\
& \quad=\nabla^{\perp} \psi \cdot \nabla \Delta \psi-\nabla^{\perp} \psi_{1} \cdot \nabla \Delta \psi_{1}, \quad \text { in } \Omega \times(0, \infty), \\
& \overline{\psi_{t}}+\nabla^{\perp} \varphi \cdot \nabla \psi-\nabla^{\perp} \varphi_{1} \cdot \nabla \psi_{1}=0, \quad \text { in } \Omega \times(0, \infty), \\
& \bar{\psi}\left(x_{1}, x_{2}, 0\right)=0, \\
& \bar{\varphi}\left(x_{1}, x_{2}, 0\right)=0
\end{aligned}
$$$$
\text { in } \Omega \times 0 \text {, }
$$$$
\bar{\psi}=0,
$$$$
\bar{\varphi}=0
$$
obtain

Similarly we have

$$
\begin{aligned}
\int_{\Omega}\left(\nabla^{\perp} \varphi_{1} \cdot \nabla \bar{\psi}\right) \bar{\psi} d x & =0 \\
\int_{\Omega} \nabla^{\perp} \bar{\varphi} \cdot \nabla\left(\Delta \varphi_{1}\right) \bar{\varphi} d x & =0 \\
\int_{\Omega}\left(\nabla^{\perp} \bar{\varphi} \cdot \nabla \bar{\psi}\right) \bar{\psi} d x & =0 \\
\int_{\Omega} \nabla^{\perp} \bar{\psi} \cdot \nabla(\Delta \bar{\psi}) \bar{\varphi} d x & =-\int_{\Omega} \Delta \bar{\psi}\left(\nabla \bar{\varphi} \cdot \nabla^{\perp} \bar{\psi}\right) d x \\
\int_{\Omega} \nabla^{\perp} \varphi_{1} \cdot \nabla(\Delta \bar{\varphi}) \bar{\varphi} d x & =-\int_{\Omega} \Delta \bar{\varphi}\left(\nabla \bar{\varphi} \cdot \nabla^{\perp} \varphi_{1}\right) d x \\
\int_{\Omega} \nabla^{\perp} \psi_{1} \cdot \nabla(\Delta \bar{\psi}) \bar{\varphi} d x & =-\int_{\Omega} \Delta \bar{\psi} \nabla \bar{\varphi} \cdot \nabla^{\perp} \psi_{1} d x \\
\int_{\Omega} \nabla^{\perp} \bar{\psi} \cdot \nabla\left(\Delta \psi_{1}\right) \bar{\varphi} d x & =-\int_{\Omega} \Delta \psi_{1} \nabla \bar{\varphi} \cdot \nabla^{\perp} \bar{\psi} d x .
\end{aligned}
$$

Notice that

$$
\overline{\psi_{t}}+\nabla^{\perp} \varphi \cdot \nabla \psi-\nabla^{\perp} \varphi_{1} \cdot \nabla \psi_{1}=0 .
$$

Multiplying equation by $\Delta \bar{\psi}$, integrating over $\Omega$, we

$$
\begin{aligned}
& \frac{1}{2} \frac{d}{d t}\|\nabla \bar{\psi}\|_{L^{2}}^{2} \\
& \quad=\int_{\Omega}\left(\nabla^{\perp} \bar{\varphi} \cdot \nabla \bar{\psi}+\nabla^{\perp} \bar{\varphi} \cdot \nabla \psi_{1}+\nabla^{\perp} \varphi_{1} \cdot \nabla \bar{\psi}\right) \Delta \bar{\psi} d x
\end{aligned}
$$

so that

Multiplying the first and second equations by $\bar{\varphi}, \bar{\psi}$, respectively, integrating over $\Omega$, we obtain

$$
\begin{aligned}
& -\frac{1}{2} \frac{d}{d t}\|\nabla \bar{\varphi}\|_{L^{2}}^{2}+\int_{\Omega} \nabla^{\perp} \bar{\varphi} \cdot \nabla(\Delta \bar{\varphi}) \bar{\varphi} d x \\
& \quad+\int_{\Omega} \nabla^{\perp} \varphi_{1} \cdot \nabla(\Delta \bar{\varphi}) \bar{\varphi} d x \\
& \quad+\int_{\Omega} \nabla^{\perp} \bar{\varphi} \cdot \nabla\left(\Delta \varphi_{1}\right) \bar{\varphi} d x=\int_{\Omega} \nabla^{\perp} \bar{\psi} \cdot \nabla(\Delta \bar{\psi}) \bar{\varphi} d x \\
& \quad+\int_{\Omega} \nabla^{\perp} \psi_{1} \cdot \nabla(\Delta \bar{\psi}) \bar{\varphi} d x+\int_{\Omega} \nabla^{\perp} \bar{\psi} \cdot \nabla\left(\Delta \psi_{1}\right) \bar{\varphi} d x, \\
& \frac{1}{2} \frac{d}{d t}\|\bar{\psi}\|_{L^{2}}^{2}+\int_{\Omega}\left(\nabla^{\perp} \bar{\varphi} \cdot \nabla \bar{\psi}\right) \bar{\psi} d x \\
& \quad+\int_{\Omega}\left(\nabla^{\perp} \bar{\varphi} \cdot \nabla \psi_{1}\right) \bar{\psi} d x+\int_{\Omega}\left(\nabla^{\perp} \varphi_{1} \cdot \nabla \bar{\psi}\right) \bar{\psi} d x=0 .
\end{aligned}
$$

It is easy to see that

$$
\begin{aligned}
\int_{\Omega} \nabla^{\perp} \bar{\varphi} \cdot \nabla(\Delta \bar{\varphi}) \bar{\varphi} d x= & \int_{\partial \Omega} \Delta \bar{\varphi}\left(\bar{\varphi} \cdot \nabla^{\perp} \bar{\varphi}\right) \cdot \vec{n} d s \\
& -\int_{\Omega} \Delta \bar{\varphi} d i v\left(\bar{\varphi} \cdot \nabla^{\perp} \bar{\varphi}\right) d x \\
& =-\int_{\Omega} \Delta \bar{\varphi} \cdot 0 d x=0 .
\end{aligned}
$$

$$
\begin{aligned}
\frac{1}{2} \frac{d}{d t}\|\nabla \bar{\varphi}\|_{L^{2}}^{2}+\frac{1}{2} \frac{d}{d t}\|\bar{\psi}\|_{L^{2}}^{2}+\frac{1}{2} \frac{d}{d t}\|\nabla \bar{\psi}\|_{L^{2}}^{2} \\
=-\int_{\Omega} \Delta \bar{\varphi}\left(\nabla \bar{\varphi} \cdot \nabla^{\perp} \varphi_{1}\right) d x+\int_{\Omega} \Delta \bar{\psi} \nabla \bar{\varphi} \cdot \nabla^{\perp} \psi_{1} d x \\
\quad+\int_{\Omega} \Delta \psi_{1} \nabla \bar{\varphi} \cdot \nabla^{\perp} \bar{\psi} d x-\int_{\Omega} \bar{\psi} \nabla \psi_{1} \cdot \nabla^{\perp} \bar{\varphi} d x \\
\quad+\int_{\Omega} \nabla^{\perp} \varphi_{1} \cdot \nabla \bar{\psi} \Delta \bar{\psi} d x+\int_{\Omega} \nabla^{\perp} \bar{\varphi} \cdot \nabla \psi_{1} \Delta \bar{\psi} d x
\end{aligned}
$$$$
=0 \text {. }
$$

It is easy to see that

$$
\begin{aligned}
\frac{1}{2} \frac{d}{d t} & \|\nabla \bar{\varphi}\|_{L^{2}}^{2}+\frac{1}{2} \frac{d}{d t}\|\bar{\psi}\|_{L^{2}}^{2} \\
& +\frac{1}{2} \frac{d}{d t}\|\nabla \bar{\psi}\|_{L^{2}}^{2}\left\|D^{2} \varphi_{1}\right\|_{L^{\infty}}\|\nabla \bar{\varphi}\|_{L^{2}}^{2} \\
& +c\left\|D^{2} \varphi_{1}\right\|_{L^{\infty}}\|\nabla \bar{\psi}\|_{L^{2}}^{2} \\
& +c\left\|\Delta \psi_{1}\right\|_{L^{\infty}}\left(\|\nabla \bar{\varphi}\|_{L^{2}}^{2}+\|\nabla \bar{\psi}\|_{L^{2}}^{2}\right) \\
& +c\left\|\nabla \psi_{1}\right\|_{L^{\infty}}\left(\|\bar{\psi}\|_{L^{2}}^{2}+\|\nabla \bar{\varphi}\|_{L^{2}}^{2}\right) \\
\leq & c\left(\|\nabla \bar{\psi}\|_{L^{2}}^{2}+\|\nabla \bar{\varphi}\|_{L^{2}}^{2}+\|\bar{\psi}\|_{L^{2}}^{2}\right) .
\end{aligned}
$$


Thanks to the Gronwall inequality, we have the following:

$$
\begin{gathered}
\|\nabla \bar{\psi}\|_{L^{2}}^{2}+\|\nabla \bar{\varphi}\|_{L^{2}}^{2}+\|\bar{\psi}\|_{L^{2}}^{2} \leq e^{c t}\left(\left\|\nabla \bar{\psi}\left(x_{1}, x_{2}, 0\right)\right\|_{L^{2}}^{2}\right. \\
\left.+\left\|\nabla \bar{\varphi}\left(x_{1}, x_{2}, 0\right)\right\|_{L^{2}}^{2}+\left\|\bar{\psi}\left(x_{1}, x_{2}, 0\right)\right\|_{L^{2}}^{2}\right)=0 .
\end{gathered}
$$

Therefore there exists a unique solution in the sense of $L^{2}\left(\left(\left(L^{2}(\Omega)\right)^{2} ; 0, T\right)\right), \forall T>0$.

\section{Analysis Stability of the Wave Solutions}

In this section we discuss the stability of the solutions, in $L^{2}(\Omega)$ for problem (36) [14, 16-18]. Firstly we could give the initial-boundary value of three conditions in Theorem 2 .

(1) $\left.\varphi\left(x_{1}, x_{2}, t\right)\right|_{t=0}=\varphi\left(a_{1} x_{1}+b_{1} x_{2}+d_{1}\right) \in H^{1}(\Omega)$, $\left.\varphi\left(x_{1}, x_{2}, t\right)\right|_{x \in \partial \Omega}=\varphi\left(a_{1} x_{1}+b_{1} x_{2}+d_{1}\right)$ $\left.\psi\left(x_{1}, x_{2}, t\right)\right|_{t=0}=\psi\left(k a_{1} x_{1}+k b_{1} x_{2}+d_{2}\right) \in H^{1}(\Omega)$, $\left.\psi\left(x_{1}, x_{2}, t\right)\right|_{x \in \partial \Omega}=\psi\left(k a_{1} x_{1}+k b_{1} x_{2}+d_{2}\right)$.

(2) $\left.\varphi\left(x_{1}, x_{2}, t\right)\right|_{t=0}=\varphi\left(d_{1}\right) \in H^{1}(\Omega),\left.\varphi\left(x_{1}, x_{2}, t\right)\right|_{x \in \partial \Omega}=$ $\varphi\left(c_{1} t+d_{1}\right)$

$\left.\psi\left(x_{1}, x_{2}, t\right)\right|_{t=0}=\psi\left(a_{2} x_{1}+b_{2} x_{2}+d_{2}\right) \in H^{1}(\Omega)$, $\left.\psi\left(x_{1}, x_{2}, t\right)\right|_{x \in \partial \Omega}=\psi\left(a_{2} x_{1}+a_{2} x_{2}+d_{2}\right)$.

(3) $\left.\varphi\left(x_{1}, x_{2}, t\right)\right|_{t=0}=-\left(c_{2} /\left(a_{1} b_{2}-a_{2} b_{1}\right)\right)\left(a_{1} x_{1}+b_{1} x_{2}+d_{1}\right) \epsilon$ $H^{1}(\Omega),\left.\varphi\left(x_{1}, x_{2}, t\right)\right|_{x \in \partial \Omega}=\varphi\left(a_{1} x_{1}+b_{1} x_{2}+c_{1} t+d_{1}\right)$ $\left.\psi\left(x_{1}, x_{2}, t\right)\right|_{t=0} \in H^{1}(\Omega),\left.\psi\left(x_{1}, x_{2}, t\right)\right|_{x \in \partial \Omega}=\psi\left(a_{2} x_{1}+\right.$ $\left.b_{2} x_{2}+c_{2} t+d_{2}\right)$.

Assume that $\Omega \subset R^{2}$ is a bounded domain. We can make the solutions $\varphi_{1}, \psi_{1}$ like the condition in Theorem 2 and satisfy $\left\|\Delta \psi_{1}\right\|_{L^{\infty}},\left\|\nabla \psi_{1}\right\|_{L^{\infty}},\left\|D^{2} \varphi_{1}\right\|_{L^{\infty}} \leq M$, respectively. Then the following problem:

$$
\begin{aligned}
& \left(\partial_{t}+u \cdot \nabla\right) w=B \cdot \nabla j, \quad \text { in } \Omega \times(0, \infty), \\
& \left(\partial_{t}+u \cdot \nabla\right) \psi=0 \quad \text { in } \Omega \times(0, \infty)
\end{aligned}
$$

has stable solution.

Assume that $\varphi, \psi$ are another solutions of the equations. Let $\varphi, \psi$ denote the solution pair of a little disturbance and let $\bar{\varphi}=\varphi-\varphi_{1}, \bar{\psi}=\psi-\psi_{1}$ be the difference of $\varphi, \varphi_{1}$, $\psi, \psi_{1}$, with initial value $\left\|\overline{\varphi_{0}}(x)\right\|_{L^{2}} \rightarrow 0,\left\|\nabla \overline{\varphi_{0}}(x)\right\|_{L^{2}} \rightarrow 0$, $\left\|\overline{\psi_{0}}(x)\right\|_{L^{2}} \rightarrow 0,\left\|\nabla \overline{\psi_{0}}(x)\right\|_{L^{2}} \rightarrow 0$ in the sense of $L^{2}(\Omega)$ and again assuming that boundary value $\varphi \rightarrow \varphi_{1}, \psi \rightarrow \psi_{1}$ in the sense of $L^{2}(\partial \Omega) \cap L^{\infty}(\partial \Omega), \forall t \in(0, T]$. Then subtracting one equation from each other in (36), we get

$$
\begin{aligned}
& \Delta \overline{\varphi_{t}}+\nabla^{\perp} \varphi \cdot \nabla \Delta \varphi-\nabla^{\perp} \varphi_{1} \cdot \nabla \Delta \varphi_{1} \\
& \quad=\nabla^{\perp} \psi \cdot \nabla \Delta \psi-\nabla^{\perp} \psi_{1} \cdot \nabla \Delta \psi_{1}, \quad \text { in } \Omega \times(0, \infty), \\
& \overline{\psi_{t}}+\nabla^{\perp} \varphi \cdot \nabla \psi-\nabla^{\perp} \varphi_{1} \cdot \nabla \psi_{1}=0, \text { in } \Omega \times(0, \infty), \\
& \bar{\psi}\left(x_{1}, x_{2}, 0\right)=\bar{\psi}_{0}\left(x_{1}, x_{2}, 0\right), \\
& \bar{\varphi}\left(x_{1}, x_{2}, 0\right)=\bar{\varphi}_{0}\left(x_{1}, x_{2}, 0\right)
\end{aligned}
$$$$
\text { in } \Omega \times 0 \text {, }
$$

$\bar{\psi}=\bar{\varphi}=h(x, t) \quad$ on $\partial \Omega \times(0, \infty)$.
Similar to uniqueness, we can obtain

$$
\begin{aligned}
\frac{1}{2} \frac{d}{d t} \| & \nabla \bar{\varphi}\left\|_{L^{2}}^{2}+\frac{1}{2} \frac{d}{d t}\right\| \bar{\psi}\left\|_{L^{2}}^{2}+\frac{1}{2} \frac{d}{d t}\right\| \nabla \bar{\psi} \|_{L^{2}}^{2} \\
= & -\int_{\Omega} \Delta \bar{\varphi}\left(\nabla \bar{\varphi} \cdot \nabla^{\perp} \varphi_{1}\right) d x+\int_{\Omega} \Delta \bar{\psi} \nabla \bar{\varphi} \cdot \nabla^{\perp} \psi_{1} d x \\
& +\int_{\Omega} \Delta \psi_{1} \nabla \bar{\varphi} \cdot \nabla^{\perp} \bar{\psi} d x-\int_{\Omega} \bar{\psi} \nabla \psi_{1} \cdot \nabla^{\perp} \bar{\varphi} d x \\
& +\int_{\Omega} \nabla^{\perp} \varphi_{1} \cdot \nabla \bar{\psi} \Delta \bar{\psi} d x+\int_{\Omega} \nabla^{\perp} \bar{\varphi} \cdot \nabla \psi_{1} \Delta \bar{\psi} d x \\
& +\int_{\partial \Omega} \bar{\varphi} \Delta \bar{\varphi} \nabla^{\perp} \bar{\varphi} \cdot \vec{n} d s+\int_{\partial \Omega} \bar{\varphi} \Delta \bar{\varphi} \nabla^{\perp} \varphi_{1} \cdot \vec{n} d s \\
& +\int_{\partial \Omega} \bar{\varphi} \Delta \varphi_{1} \nabla^{\perp} \bar{\varphi} \cdot \vec{n} d s-\int_{\partial \Omega} \bar{\varphi} \Delta \bar{\psi} \nabla^{\perp} \bar{\psi} \cdot \vec{n} d s \\
& -\int_{\partial \Omega} \bar{\varphi} \Delta \bar{\psi} \nabla^{\perp} \bar{\psi} \cdot \vec{n} d s-\int_{\partial \Omega} \bar{\varphi} \Delta \psi_{1} \nabla^{\perp} \bar{\psi} \cdot \vec{n} d s \\
& -\frac{1}{2} \int_{\partial \Omega} \bar{\psi} \nabla^{\perp} \bar{\varphi} \cdot \vec{n} d s .
\end{aligned}
$$

It is easy to see that

$$
\begin{aligned}
& \frac{1}{2} \frac{d}{d t}\|\nabla \bar{\varphi}\|_{L^{2}}^{2}+\frac{1}{2} \frac{d}{d t}\|\bar{\psi}\|_{L^{2}}^{2}+\frac{1}{2} \frac{d}{d t}\|\nabla \bar{\psi}\|_{L^{2}}^{2} \leq c\left(\|\nabla \bar{\psi}\|_{L^{2}}^{2}\right. \\
& \left.\quad+\|\nabla \bar{\varphi}\|_{L^{2}}^{2}+\|\bar{\psi}\|_{L^{2}}^{2}\right) \\
& \quad+c_{0}\left(\|\bar{\varphi}\|_{L^{2}(\partial \Omega)}\left\|\nabla^{\perp} \bar{\varphi}\right\|_{L^{2}(\partial \Omega)}\|\Delta \bar{\varphi}\|_{L^{\infty}(\partial \Omega)}\right. \\
& \quad+\|\bar{\varphi}\|_{L^{2}(\partial \Omega)}\left\|\nabla^{\perp} \bar{\varphi}\right\|_{L^{2}(\partial \Omega)}\|\Delta \bar{\varphi}\|_{L^{\infty}(\partial \Omega)} \\
& \quad+\|\bar{\varphi}\|_{L^{2}(\partial \Omega)}\left\|\nabla^{\perp} \bar{\varphi}\right\|_{L^{2}(\partial \Omega)}\left\|\Delta \varphi_{1}\right\|_{L^{\infty}(\partial \Omega)} \\
& \quad+\|\bar{\varphi}\|_{L^{2}(\partial \Omega)}\left\|\nabla^{\perp} \bar{\psi}\right\|_{L^{2}(\partial \Omega)}\|\Delta \bar{\psi}\|_{L^{\infty}(\partial \Omega)} \\
& \quad+\|\bar{\varphi}\|_{L^{2}(\partial \Omega)}\left\|\nabla^{\perp} \psi_{1}\right\|_{L^{2}(\partial \Omega)}\|\Delta \bar{\psi}\|_{L^{\infty}(\partial \Omega)} \\
& \left.\quad+\|\bar{\varphi}\|_{L^{2}(\partial \Omega)}\left\|\nabla^{\perp} \bar{\psi}\right\|_{L^{2}(\partial \Omega)}\left\|\Delta \psi_{1}\right\|_{L^{\infty}(\partial \Omega)}\|\bar{\psi}\|_{L^{\infty}(\partial \Omega)}\|\|_{L^{2}(\partial \Omega)}\left\|\nabla^{\perp} \bar{\varphi}\right\|_{L^{2}(\partial \Omega)}\|\bar{\psi}\|_{L^{\infty}(\partial \Omega)}\right) . \\
& \quad+\|\bar{\psi}\|_{L^{2}(\partial \Omega)}\left\|\nabla^{\perp} \varphi_{1}\right\|_{L^{2}(\partial \Omega)}
\end{aligned}
$$

Since $\varphi \rightarrow \varphi_{1}, \psi \rightarrow \psi_{1}$ in the sense of $L^{2}(\partial \Omega) \bigcap L^{\infty}(\partial \Omega)$, we can make, for every $\epsilon>0$,

$$
\begin{aligned}
& \left(\|\bar{\varphi}\|_{L^{2}(\partial \Omega)}\left\|\nabla^{\perp} \bar{\varphi}\right\|_{L^{2}(\partial \Omega)}\|\Delta \bar{\varphi}\|_{L^{\infty}(\partial \Omega)}\right. \\
& \quad+\|\bar{\varphi}\|_{L^{2}(\partial \Omega)}\left\|\nabla^{\perp} \overline{\varphi_{1}}\right\|_{L^{2}(\partial \Omega)}\|\Delta \bar{\varphi}\|_{L^{\infty}(\partial \Omega)} \\
& \quad+\|\bar{\varphi}\|_{L^{2}(\partial \Omega)}\left\|\nabla^{\perp} \bar{\varphi}\right\|_{L^{2}(\partial \Omega)}\left\|\Delta \varphi_{1}\right\|_{L^{\infty}(\partial \Omega)} \\
& \quad+\|\bar{\varphi}\|_{L^{2}(\partial \Omega)}\left\|\nabla^{\perp} \bar{\psi}\right\|_{L^{2}(\partial \Omega)}\|\Delta \bar{\psi}\|_{L^{\infty}(\partial \Omega)}
\end{aligned}
$$




$$
\begin{aligned}
& +\|\bar{\varphi}\|_{L^{2}(\partial \Omega)}\left\|\nabla^{\perp} \psi_{1}\right\|_{L^{2}(\partial \Omega)}\|\Delta \bar{\psi}\|_{L^{\infty}(\partial \Omega)} \\
& +\|\bar{\varphi}\|_{L^{2}(\partial \Omega)}\left\|\nabla^{\perp} \bar{\psi}\right\|_{L^{2}(\partial \Omega)}\left\|\Delta \psi_{1}\right\|_{L^{\infty}(\partial \Omega)} \\
& +\|\bar{\psi}\|_{L^{2}(\partial \Omega)}\left\|\nabla^{\perp} \bar{\varphi}\right\|_{L^{2}(\partial \Omega)}\|\bar{\psi}\|_{L^{\infty}(\partial \Omega)} \\
& \left.+\|\bar{\psi}\|_{L^{2}(\partial \Omega)}\left\|\nabla^{\perp} \varphi_{1}\right\|_{L^{2}(\partial \Omega)}\|\bar{\psi}\|_{L^{\infty}(\partial \Omega)}\right) \leq \epsilon .
\end{aligned}
$$
$(0, T]$,

Using the Gronwall inequality in (*), for every $\forall t \in$

$$
\begin{aligned}
& \|\nabla \bar{\psi}\|_{L^{2}}^{2}+\|\nabla \bar{\varphi}\|_{L^{2}}^{2}+\|\bar{\psi}\|_{L^{2}}^{2} \leq e^{c t}\left(\left\|\nabla \bar{\psi}\left(x_{1}, x_{2}, 0\right)\right\|_{L^{2}}^{2}\right. \\
& \left.\quad+\left\|\nabla \bar{\varphi}\left(x_{1}, x_{2}, 0\right)\right\|_{L^{2}}^{2}+\left\|\bar{\psi}\left(x_{1}, x_{2}, 0\right)\right\|_{L^{2}}^{2}\right)+2 \epsilon e^{c t} \\
& \quad \longrightarrow 0
\end{aligned}
$$

As $\varphi \rightarrow \varphi_{1}, \psi \rightarrow \psi_{1}$ in the sense of $L^{2}(\partial \Omega) \bigcap L^{\infty}(\partial \Omega)$ and $\left\|\overline{\varphi_{0}}(x)\right\|_{L^{2}} \rightarrow 0,\left\|\nabla \overline{\varphi_{0}}(x)\right\|_{L^{2}} \rightarrow 0,\left\|\overline{\psi_{0}}(x)\right\|_{L^{2}} \rightarrow 0$, $\left\|\nabla \overline{\psi_{0}}(x)\right\|_{L^{2}} \rightarrow 0$, in the sense of $L^{2}(\Omega)$. So we reach the stability of the solution in the finite time.

\section{The Lyapunov Stability of Steady State Solution}

In this section we discuss the stability of the steady state solutions, in $L^{2}(\Omega)$ for problem (41).

Definition 4. A steady state solution $\bar{v}$ is said to be stable if and only if $\bar{v}$ in any one of the neighborhood $V$, there is a neighborhood $W$ of $\bar{v}$, making any solutions $v(t, \cdot)$ with the initial condition $v(0, \cdot) \in W$ satisfy $v(t, \cdot) \in V(\forall t \geq 0)$.

Assume that $\Omega \subset R^{2}$ is a bounded domain. We can make the solutions $\varphi_{1}, \psi_{1}$ like the condition in Theorem 2 and satisfy $\left\|\Delta \psi_{1}\right\|_{L^{\infty}},\left\|\nabla \psi_{1}\right\|_{L^{\infty}},\left\|D^{2} \varphi_{1}\right\|_{L^{\infty}} \leq M$, respectively. Then the following problem solutions:

$$
\begin{aligned}
& \left(\partial_{t}+u \cdot \nabla\right) w=B \cdot \nabla j, \quad \text { in } \Omega \times(0, \infty), \\
& \left(\partial_{t}+u \cdot \nabla\right) \psi=0 \quad \text { in } \Omega \times(0, \infty)
\end{aligned}
$$

are Lyapunov stable.

Assume that $\varphi, \psi$ are another solutions of the equations. Let $\varphi, \psi$ denote the solution pair of a little disturbance and let $\bar{\varphi}=\varphi-\varphi_{1}, \bar{\psi}=\psi-\psi_{1}$ be the difference of $\varphi, \varphi_{1}$, $\psi, \psi_{1}$, with initial value $\left\|\overline{\varphi_{0}}(x)\right\|_{L^{2}} \rightarrow 0,\left\|\nabla \overline{\varphi_{0}}(x)\right\|_{L^{2}} \rightarrow 0$, $\left\|\overline{\psi_{0}}(x)\right\|_{L^{2}} \rightarrow 0,\left\|\nabla \overline{\psi_{0}}(x)\right\|_{L^{2}} \rightarrow 0$, in the sense of $L^{2}(\Omega)$ and assume that boundary value $\varphi \rightarrow \varphi_{1}, \psi \rightarrow \psi_{1}$ in the sense of $L^{2}(\partial \Omega) \cap L^{\infty}(\partial \Omega), \forall t \in(0, T]$. Then subtracting one equation from each other in (41), we get

$$
\begin{aligned}
\Delta \overline{\varphi_{t}} & +\nabla^{\perp} \varphi \cdot \nabla \Delta \varphi-\nabla^{\perp} \varphi_{1} \cdot \nabla \Delta \varphi_{1} \\
& =\nabla^{\perp} \psi \cdot \nabla \Delta \psi-\nabla^{\perp} \psi_{1} \cdot \nabla \Delta \psi_{1}, \quad \text { in } \Omega \times(0, \infty),
\end{aligned}
$$

$$
\begin{aligned}
& \overline{\psi_{t}}+\nabla^{\perp} \varphi \cdot \nabla \psi-\nabla^{\perp} \varphi_{1} \cdot \nabla \psi_{1}=0, \quad \text { in } \Omega \times(0, \infty), \\
& \bar{\psi}\left(x_{1}, x_{2}, 0\right)=\bar{\psi}_{0}\left(x_{1}, x_{2}, 0\right), \\
& \bar{\varphi}\left(x_{1}, x_{2}, 0\right)=\bar{\varphi}_{0}\left(x_{1}, x_{2}, 0\right)
\end{aligned}
$$$$
\text { in } \Omega \times 0 \text {. }
$$

Similar to uniqueness, we can obtain

$$
\begin{aligned}
\frac{1}{2} \frac{d}{d t} \| & \nabla \bar{\varphi}\left\|_{L^{2}}^{2}+\frac{1}{2} \frac{d}{d t}\right\| \bar{\psi}\left\|_{L^{2}}^{2}+\frac{1}{2} \frac{d}{d t}\right\| \nabla \bar{\psi} \|_{L^{2}}^{2} \\
= & -\int_{\Omega} \Delta \bar{\varphi}\left(\nabla \bar{\varphi} \cdot \nabla^{\perp} \varphi_{1}\right) d x+\int_{\Omega} \Delta \bar{\psi} \nabla \bar{\varphi} \cdot \nabla^{\perp} \psi_{1} d x \\
& +\int_{\Omega} \Delta \psi_{1} \nabla \bar{\varphi} \cdot \nabla^{\perp} \bar{\psi} d x-\int_{\Omega} \bar{\psi} \nabla \psi_{1} \cdot \nabla^{\perp} \bar{\varphi} d x \\
& +\int_{\Omega} \nabla^{\perp} \varphi_{1} \cdot \nabla \bar{\psi} \Delta \bar{\psi} d x+\int_{\Omega} \nabla^{\perp} \bar{\varphi} \cdot \nabla \psi_{1} \Delta \bar{\psi} d x \\
& +\int_{\partial \Omega} \bar{\varphi} \Delta \bar{\varphi} \nabla^{\perp} \bar{\varphi} \cdot \vec{n} d s+\int_{\partial \Omega} \bar{\varphi} \Delta \bar{\varphi} \nabla^{\perp} \varphi_{1} \cdot \vec{n} d s \\
& +\int_{\partial \Omega} \bar{\varphi} \Delta \varphi_{1} \nabla^{\perp} \bar{\varphi} \cdot \vec{n} d s-\int_{\partial \Omega} \bar{\varphi} \Delta \bar{\psi} \nabla^{\perp} \bar{\psi} \cdot \vec{n} d s \\
& -\int_{\partial \Omega} \bar{\varphi} \Delta \bar{\psi} \nabla^{\perp} \bar{\psi} \cdot \vec{n} d s-\int_{\partial \Omega} \bar{\varphi} \Delta \psi_{1} \nabla^{\perp} \bar{\psi} \cdot \vec{n} d s \\
& -\frac{1}{2} \int_{\partial \Omega} \bar{\psi} \nabla^{\perp} \bar{\varphi} \cdot \vec{n} \bar{\psi} d s .
\end{aligned}
$$

It is easy to see that

$$
\begin{aligned}
& \frac{1}{2} \frac{d}{d t}\|\nabla \bar{\varphi}\|_{L^{2}}^{2}+\frac{1}{2} \frac{d}{d t}\|\bar{\psi}\|_{L^{2}}^{2}+\frac{1}{2} \frac{d}{d t}\|\nabla \bar{\psi}\|_{L^{2}}^{2} \\
& \quad \leq c\left(\|\nabla \bar{\psi}\|_{L^{2}}^{2}+\|\nabla \bar{\varphi}\|_{L^{2}}^{2}+\|\bar{\psi}\|_{L^{2}}^{2}\right) \\
& \quad+c_{0}\left(\|\bar{\varphi}\|_{L^{2}(\partial \Omega)}\left\|\nabla^{\perp} \bar{\varphi}\right\|_{L^{2}(\partial \Omega)}\|\Delta \bar{\varphi}\|_{L^{\infty}(\partial \Omega)}\right. \\
& \quad+\|\bar{\varphi}\|_{L^{2}(\partial \Omega)}\left\|\nabla^{\perp} \bar{\varphi}\right\|_{L^{2}(\partial \Omega)}\|\Delta \bar{\varphi}\|_{L^{\infty}(\partial \Omega)} \\
& \quad+\|\bar{\varphi}\|_{L^{2}(\partial \Omega)}\left\|\nabla^{\perp} \bar{\varphi}\right\|_{L^{2}(\partial \Omega)}\left\|\Delta \varphi_{1}\right\|_{L^{\infty}(\partial \Omega)} \\
& \quad+\|\bar{\varphi}\|_{L^{2}(\partial \Omega)}\left\|\nabla^{\perp} \bar{\psi}\right\|_{L^{2}(\partial \Omega)}\|\Delta \bar{\psi}\|_{L^{\infty}(\partial \Omega)} \\
& \quad+\|\bar{\varphi}\|_{L^{2}(\partial \Omega)}\left\|\nabla^{\perp} \psi\right\|_{L^{2}(\partial \Omega)}\|\Delta \bar{\psi}\|_{L^{\infty}(\partial \Omega)} \\
& \quad+\|\bar{\varphi}\|_{L^{2}(\partial \Omega)}\left\|\nabla^{\perp} \bar{\psi}\right\|_{L^{2}(\partial \Omega)}\|\Delta \psi\|_{L^{\infty}(\partial \Omega)} \\
& \quad+\|\bar{\psi}\|_{L^{2}(\partial \Omega)}\left\|\nabla^{\perp} \bar{\varphi}\right\|_{L^{2}(\partial \Omega)}\|\bar{\psi}\|_{L^{\infty}(\partial \Omega)} \\
& \left.\quad+\|\bar{\psi}\|_{L^{2}(\partial \Omega)}\left\|\nabla^{\perp} \varphi_{1}\right\|_{L^{2}(\partial \Omega)}\|\bar{\psi}\|_{L^{\infty}(\partial \Omega)}\right)
\end{aligned}
$$

Since $\varphi \rightarrow \varphi_{1}, \psi \rightarrow \psi_{1}$ in the sense of $L^{2}(\partial \Omega) \bigcap L^{\infty}(\partial \Omega)$, we can make, for every $\epsilon>0$,

$$
\begin{aligned}
& \left(\|\bar{\varphi}\|_{L^{2}(\partial \Omega)}\left\|\nabla^{\perp} \bar{\varphi}\right\|_{L^{2}(\partial \Omega)}\|\Delta \bar{\varphi}\|_{L^{\infty}(\partial \Omega)}\right. \\
& \quad+\|\bar{\varphi}\|_{L^{2}(\partial \Omega)}\left\|\nabla^{\perp} \overline{\varphi_{1}}\right\|_{L^{2}(\partial \Omega)}\|\Delta \bar{\varphi}\|_{L^{\infty}(\partial \Omega)}
\end{aligned}
$$




$$
\begin{aligned}
& +\|\bar{\varphi}\|_{L^{2}(\partial \Omega)}\left\|\nabla^{\perp} \bar{\varphi}\right\|_{L^{2}(\partial \Omega)}\left\|\Delta \varphi_{1}\right\|_{L^{\infty}(\partial \Omega)} \\
& +\|\bar{\varphi}\|_{L^{2}(\partial \Omega)}\left\|\nabla^{\perp} \bar{\psi}\right\|_{L^{2}(\partial \Omega)}\|\Delta \bar{\psi}\|_{L^{\infty}(\partial \Omega)} \\
& +\|\bar{\varphi}\|_{L^{2}(\partial \Omega)}\left\|\nabla^{\perp} \psi_{1}\right\|_{L^{2}(\partial \Omega)}\|\Delta \bar{\psi}\|_{L^{\infty}(\partial \Omega)} \\
& +\|\bar{\varphi}\|_{L^{2}(\partial \Omega)}\left\|\nabla^{\perp} \bar{\psi}\right\|_{L^{2}(\partial \Omega)}\left\|\Delta \psi_{1}\right\|_{L^{\infty}(\partial \Omega)} \\
& +\|\bar{\psi}\|_{L^{2}(\partial \Omega)}\left\|\nabla^{\perp} \bar{\varphi}\right\|_{L^{2}(\partial \Omega)}\|\bar{\psi}\|_{L^{\infty}(\partial \Omega)} \\
& \left.+\|\bar{\psi}\|_{L^{2}(\partial \Omega)}\left\|\nabla^{\perp} \varphi_{1}\right\|_{L^{2}(\partial \Omega)}\|\bar{\psi}\|_{L^{\infty}(\partial \Omega)}\right) \leq \epsilon .
\end{aligned}
$$

Using the Gronwall inequality in $(* *)$, for every $\forall t \in$ $(0, T]$,

$$
\begin{aligned}
& \|\nabla \bar{\psi}\|_{L^{2}}^{2}+\|\nabla \bar{\varphi}\|_{L^{2}}^{2}+\|\bar{\psi}\|_{L^{2}}^{2} \leq e^{c t}\left(\left\|\nabla \bar{\psi}\left(x_{1}, x_{2}, 0\right)\right\|_{L^{2}}^{2}\right. \\
& \left.\quad+\left\|\nabla \bar{\varphi}\left(x_{1}, x_{2}, 0\right)\right\|_{L^{2}}^{2}+\left\|\bar{\psi}\left(x_{1}, x_{2}, 0\right)\right\|_{L^{2}}^{2}\right)+2 \epsilon e^{c t} \\
& \quad \longrightarrow 0
\end{aligned}
$$

As $\varphi \rightarrow \varphi_{1}, \psi \rightarrow \psi_{1}$ in the sense of $L^{2}(\partial \Omega) \bigcap L^{\infty}(\partial \Omega)$ and $\left\|\overline{\varphi_{0}}(x)\right\|_{L^{2}} \rightarrow 0,\left\|\nabla \overline{\varphi_{0}}(x)\right\|_{L^{2}} \rightarrow 0,\left\|\overline{\psi_{0}}(x)\right\|_{L^{2}} \rightarrow 0$, $\left\|\nabla \overline{\psi_{0}}(x)\right\|_{L^{2}} \rightarrow 0$, in the sense of $L^{2}(\Omega)$. Definition 4 is satisfied. So we reach the Lyapunov stability of the steady state solutions in finite time.

\section{Conclusions}

In this paper, we studied the symmetry groups by using the classical Lie Group method to structural equation solutions. First, we perform Lie symmetry analysis for the MHD equation and get its infinitesimal generator. Then, we obtain many solutions by it. It can be seen by the results of this paper that the Lie Group method is an effective method for studying nonlinear partial differential equations.

\section{Competing Interests}

The authors declare that they have no competing interests.

\section{Authors' Contributions}

All authors contributed equally to the writing of this paper. All authors read and approved the final manuscript.

\section{Acknowledgments}

The first author is grateful to Professor Ganshan Yang and Yangrong Li for their support and also thanks Jiali Yu for help in writing this paper. The work is supported by the National Natural Science Foundation of China (nos. 11161057 and 11561076).

\section{References}

[1] D. Cordoba and C. Marliani, "Evolution of current sheets and regularity of ideal incompressible magnetic fluids in 2D," Communications on Pure and Applied Mathematics, vol. 53, no. 4, pp. 512-524, 2000.

[2] D. Biskamp, Nonlinear Magnetohydrodynamics, vol. 1 of Cambridge Monographs on Plasma Physics, Cambridge University Press, Cambridge, Uk, 1993.

[3] Q. L. Chen, C. X. Miao, and Z. F. Zhang, "The Beale-KatoMajda criterion for the 3D magneto-hydrodynamics equations," Communications in Mathematical Physics, vol. 275, no. 3, pp. 861-872, 2007.

[4] J. Wu, "Viscous and inviscid magnetohydrodynamics equations," Journal d'Analyse Mathématique, vol. 73, pp. 251-265, 1997.

[5] C. Cao and J. Wu, "Global regularity for the 2D MHD equations with mixed partial dissipation and magnetic diffusion," https://arxiv.org/abs/0901.2908.

[6] C. He and Z. Xin, "Partial regularity of suitable weak solutions to the incompressible magnetohydrodynamic equations," Journal of Functional Analysis, vol. 227, no. 1, pp. 113-152, 2005.

[7] V. Vyalov, "Partial regularity of solutions to the magnetohydrodynamic equations," Journal of Mathematical Sciences (New York), vol. 150, no. 1, pp. 1771-1786, 2008.

[8] M. Sermange and R. Temam, "Some mathematical questions related to the MHD equations," Communications on Pure and Applied Mathematics, vol. 36, no. 5, pp. 635-664, 1983.

[9] P. J. Olver, Applications of Lie Groups to Differential Equations, vol. 107, Springer, New York, NY, USA, 2nd edition, 1993.

[10] S. Yang and C. Hua, "Lie symmetry reductions and exact solutions of a coupled KdV-Burgers equation," Applied Mathematics and Computation, vol. 234, pp. 579-583, 2014.

[11] S. Shen, C. Qu, and Q. Huang, "Lie group classification of the $\mathrm{N}$-th-order nonlinear evolution equations," Science China Mathematics, vol. 54, no. 12, pp. 2553-2572, 2011.

[12] E. Casella, P. Secchi, and P. Trebeschi, "Global classical solutions for MHD system," Journal of Mathematical Fluid Mechanics, vol. 5, no. 1, pp. 70-91, 2003.

[13] X. Liao, The Stability of the Theory, Method and Application, Huazhong University of Science and Technology Press, 2nd edition, 2010.

[14] W. Song, H. Li, G. Yang, and G. X. Yuan, "Nonhomogeneous boundary value problem for $(I, J)$ similar Solutions of incompressible two-dimensional Euler equations," Journal of Inequalities and Applications, vol. 2014, no. 1, article 277, 2014.

[15] M. D. Gunzburger, A. J. Meir, and J. S. Peterson, "On the existence, uniqueness, and finite element approximation of solutions of the equations of stationary, incompressible magnetohydrodynamics," Mathematics of Computation, vol. 56, no. 194, pp. 523-563, 1991.

[16] L.-J. Yang and J.-M. Wang, "Staility of a damped hyperbolic Timoshenko system coupled with a heat equation," Asian Journal of Control, vol. 16, no. 2, pp. 546-555, 2014.

[17] G. Yang, $\delta$-Viscosity solution, blow up solution and global solution of multidimensional Landau-Lifshitz equations [Ph.D. thesis], China Academy of Engineering Physics, Sichuan, China, 2002.

[18] V. I. Yudovitch, "Non-stationary flow of an ideal incompressible liquid," USSR Computational Mathematics and Mathematical Physics, vol. 3, no. 6, pp. 1407-1456, 1963. 


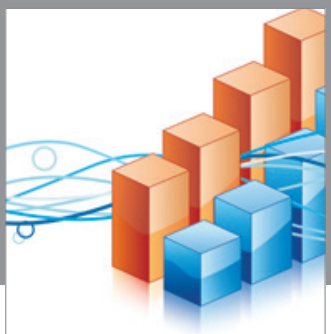

Advances in

Operations Research

vatem alat4

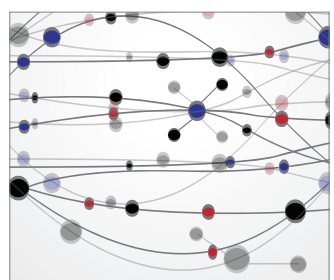

\section{The Scientific} World Journal
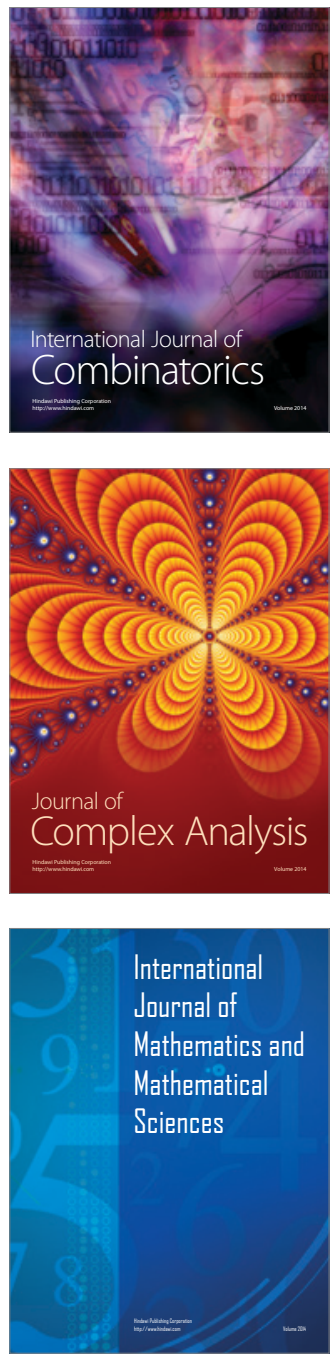
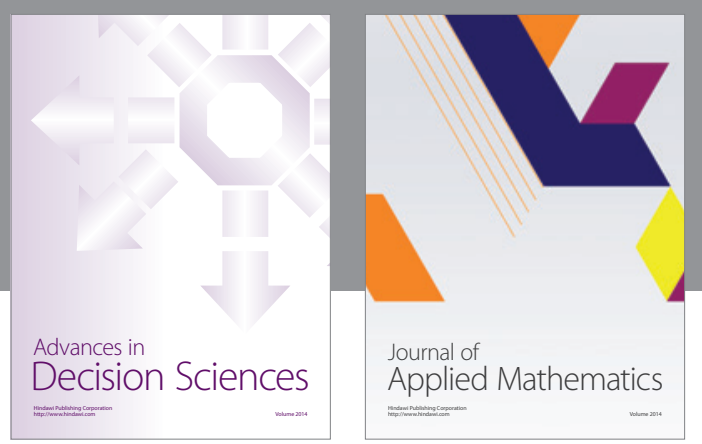

Algebra

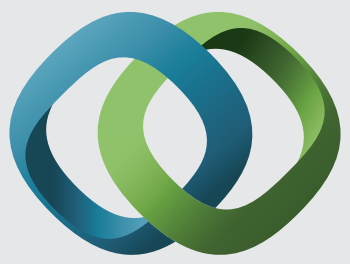

\section{Hindawi}

Submit your manuscripts at

http://www.hindawi.com
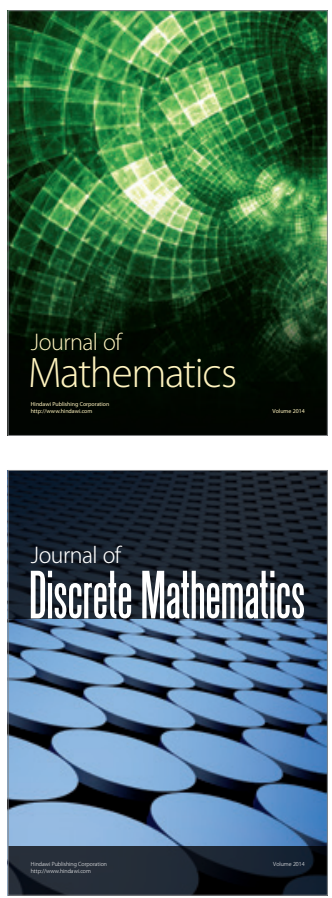

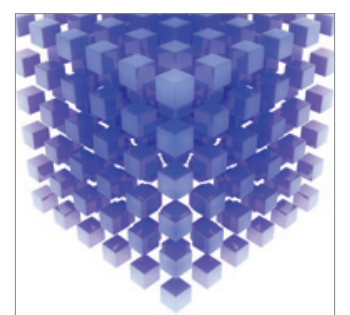

Mathematical Problems in Engineering
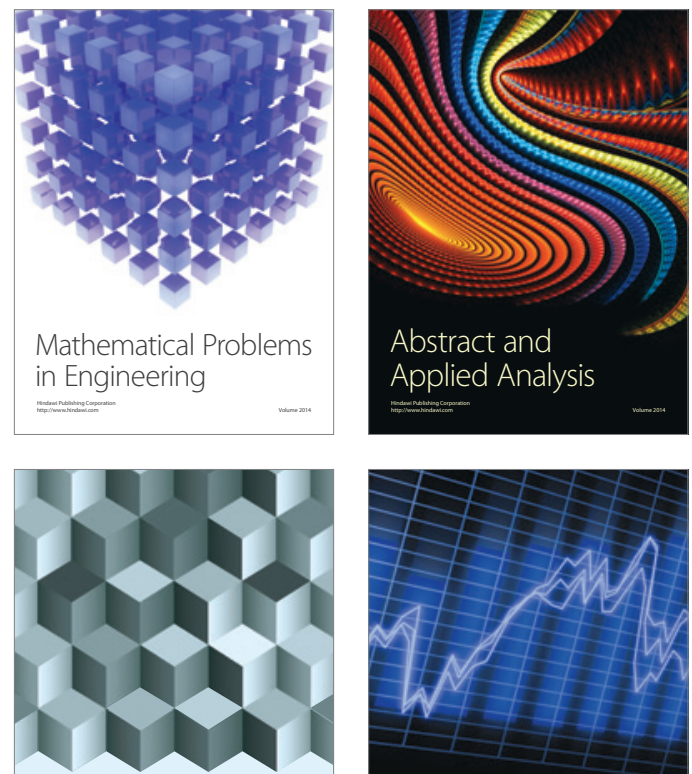

Journal of

Function Spaces

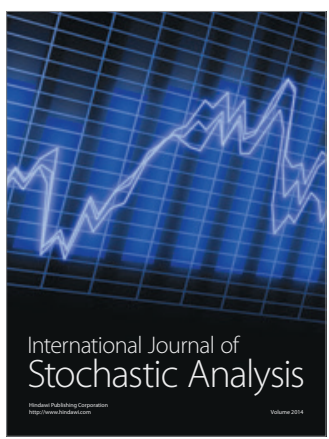

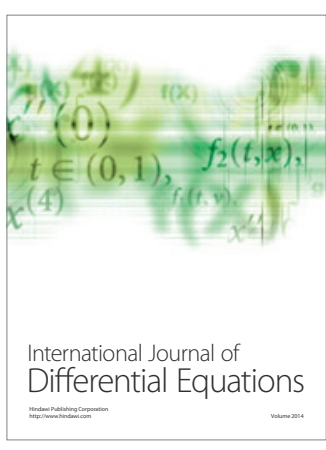
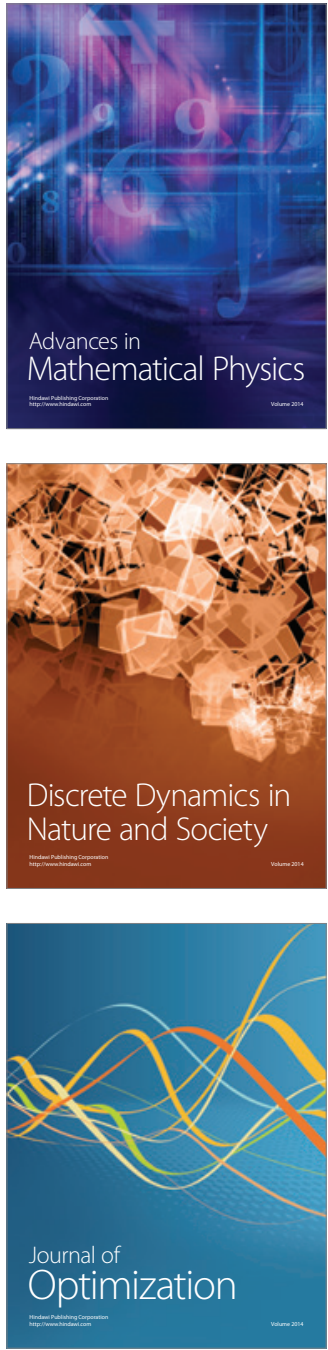